\title{
ACEDR: Automatic Compiler Error Detection and Recovery for COTS CPU and Caches
}

\author{
Y. Nezzari (i) and C. P. Bridges (1)
}

\begin{abstract}
Recently there has been an increasing demand for more powerful processors for the next-generation space missions, such as communication and earth observation. The challenge is how to improve the reliability of the processor under the "single event effects" in orbit. We have previously proposed a new way of implementing any traditional software error detection and correction techniques at instruction level, capable of covering both the CPU and caches of "commercial off the shelf" processors. In this paper, a novel way of evaluation of the software protection is presented, based on a theoretical model and software injection experiments to predict the reliability of the whole processing architecture. The fault injection will evaluate the ability of the protection code to detect and recover errors in addition to the accuracy of the reliability models, by comparing the reliability of the theoretical predictions to the reliability of the injection experiments. Automatic compiler error detection and recovery improves the reliability of the system by reducing the error rate of "single event upsets." In some benchmarks, the error rate was reduced to less than $1 \%$. This research has been tested in two machines; Intel core i5-3470 and a Raspberry Pi 3. On the first processor, the overhead was less than $15 \%$, and on the second one, the overhead was less than $17 \%$. This research can also be ported to multiple high level languages, with the ability to cover multiple instructions and datatypes.
\end{abstract}

Index Terms-Cache, compiler, CPU, error detection and recovery, processing architecture, reliability, single event upsets (SEUs).

\section{INTRODUCTION}

I N RECENT years, dramatic technology scaling in integrated circuits (ICs) is occurring. The scaling comes with smaller and faster transistors, enabling higher transistor counts that led to higher performing processing architectures with low power consumption, less size, and at a fraction of the cost. However, these new technologies are sensitive to noise margins with low threshold voltages that give rise to transient effects caused by environmental or external factors, such as background or cosmic radiation effects. Another disturbance source is internal intermittent effects specific to the IC, such as when operating conditions change (e.g., temperature, component wear out, component

Manuscript received July 5, 2018; revised December 25, 2018 and April 7, 2019; accepted June 15, 2019. Date of publication July 18, 2019; date of current version August 29, 2019. This work was supported by in part by the Algerian Space Agency, in part by the U.K. Space Agency, and in part by the Surrey Space Centre at the University of Surrey. Associate Editor: F. Belli. (Corresponding author: Yasser Nezzari.)

The authors are with the Surrey Space Centre at the University of Surrey, GU27XH Guildford, U.K. (e-mail: y.nezzari@surrey.ac.uk; c.p.bridges@ surrey.ac.uk).

Color versions of one or more of the figures in this paper are available online at http://ieeexplore.ieee.org.

Digital Object Identifier 10.1109/TR.2019.2925086 overload) [1]-[4]. These are often soft errors and do not cause permanent damage. As transistors become increasingly prone to soft errors, high reliability should not be exclusive to mission critical processing purposes, but should also be extended to processors used in mainstream computing and embedded architectures.

For mission critical domains like the space, radiation hardened (RH) and RH by design (RHBD) processing architectures are developed for their high reliability in a harsh environment. Even with their efficiency in mitigating single event effects (SEEs), RH and RHBD still lag behind in terms of performance, compared to their commercial off the shelf (COTS) relatives. The performance gap is estimated to be from five to ten years [5]. In addition, RH and RHBD are costly and consume higher power. All the previous limitations have steered new space developers to consider the use of COTS.

To mitigate errors, hardware redundancy can detect and recover SEEs [6], [7]; however, it is only suitable for the domains that have no budget restrictions. An example of the hard redundancy is the use of hard error correction codes (ECC) that could be costly in terms of both performance and power [8], [9]. Hard redundancy can be impractical in embedded systems due to power constraints. Software error detection and recovery techniques are more appealing to tackle the problem of soft errors because of their flexible implementation and low cost. Soft error detection and recovery can be applied on COTS, allowing designers to have an order of magnitude in performance compared to the hard redundancy.

The current trend in the implementation of soft-protection techniques still lags in terms of performance and ability to detect and correct errors. The lag in performance is due to the inefficiency in using the memory and CPU abundant resources of the processing architecture leading to a bottleneck. Most of the techniques applied to detect and recover errors only cover the CPU registers; however most of literature ignores the protection of the memory system of the processing architecture, because of the assumption that the memory has hard-ECC protection. In this paper, we demonstrate that Read/Write (R/W) operations from/to the memory have as much importance as the rest of instructions in the study of reliability.

In this paper, we propose "automatic compiler error detection and recovery" (ACEDR), an original software error detection and recovery technique, for automatically applying protection code in the LLVM compiler framework. The applied codes are capable of automatically detecting and recovering soft errors at runtime. This work is based on two LLVM passes: An analysis 
and a transformation. The analysis pass will be executed on the intermediate representation (IR) code intended to be protected and provides the statistics and information about memory instruction dependencies. The transformation pass will add the protection code by adding redundant instructions and calling a voter function to detect and recover at runtime. The voter works as in triple modular redundancy (TMR). We will show in this paper the importance of protecting both the memory (R/W) instructions and CPU instructions (Arithmetic and logic operators, and branching) on the reliability predictions outcome. We start by studying the different error rates reductions of using partial protection techniques, where we start by only protecting CPU instructions and then memory instructions, and then combining both-following on from our previous work [10]. Using instruction level redundancy will allow for the mitigation of one or more bit errors and could tolerate single event upsets (SEUs) and multiple bit upsets. Our paper contributes to the state of the art with the following points.

1) Low overhead compared to the state of the art, less than $15 \%$ in Intel core $15-3470$ and a less than $17 \%$ in Raspberry Pi 3.

2) A reliability prediction model that predicts the reliabilities of all the processing architecture components, and to quantify the reliability added using the software protection code.

3) High error detection and recovery from a new injector where error rates can be reduced to less than $1 \%$ in some benchmarks.

4) Multiple data and instruction types (i32, i32 $, \mathrm{i} 1, \mathrm{i} 8, \mathrm{i} 8^{\star}$, $\mathrm{i} 64$, float and double, float pointers and double pointers) [11] have been protected, after [10] was extended, in addition to both the CPU and the memory R/W instructions types.

5) Comparison of the reliability predictions with the reliability obtained from the injection experiment of the protected code.

The rest of the paper is organized as follows. In Section II, the solutions that have been applied to overcome the SEEs obstacle will be introduced, focusing on the soft-based protection techniques. In Section III, the reliability prediction equations will be derived for the different processing component (CPU, Caches, and RAM), in the unprotected and the protected modes. In Section IV, the implementation of ACEDR will be explained, including how the compiler's optimizer has been altered to add different protection techniques. In Section $\mathrm{V}$, the fault injection experiment will be explained, and the different error rates reduction from the unprotected to the protected codes will be shown. In Section VI, the predictions of reliability will be compared to the reliability obtained from the injection experiment. Section VII concludes this paper.

\section{FAult TOLERANCE}

A system is categorized as fault tolerant if it always produces a correct outcome, even if its components were corrupted. To ensure correctness, the fault tolerant system must detect and correct errors before their propagation to the external state. Concurrency can be used for error detection. For the recovery, re-execution, rollback recovery, roll forward recovery, and check pointing [12] can be used. Fault tolerance can be achieved either by masking errors or detection and recovery [12].

\section{A. Fault Tolerance by Redundancy}

Redundancy in software or hardware can be used for fault tolerance. Hardware errors can be detected and recovered by adding extra memory or processing units. In software, programs can be replicated entirely or partially. N-modular redundancy (NMR) is a widely used scheme for error detection and/or recovery, where $\mathrm{N}$ represents the number of identical cloned processing elements, with the same data.

Dual modular redundancy (DMR) is an NMR scheme with two identical elements, the original and a replicated one [13]. DMR is not able to recover error, but using its comparator enables it to detect errors. On the other hand, TMR has three redundant elements, enabling it to detect and recover errors using majority voting logic. In terms of reliability, TMR has a higher rate than DMR because of its extra element allowing it not only to detect errors, but also to recover from them. The downside of TMR compared to DMR is its performance, DMR is just detecting so it takes less time than the TMR. In general, there are three classes of redundancy:

1) the addition of redundant hardware elements allowing the same tasks on various components, known as the spatial redundancy;

2) the implementation of NMR schemes on the same hardware, where $N$ redundant similar operations are executed sequentially, known as temporal redundancy;

3) replication of data and CPU registers can be achieved by computing extra check bits of the original data, using error detection codes or ECC. This is known as the class of information redundancy.

\section{B. Software-Based Fault Tolerance and Redundancy Approaches}

1) Process-Level Replication (PLR): PLR's implementation makes changes at kernel level. This technique uses the redundancy of maestro processing architecture [14] to execute the same application on three different cores. TMR is used to decide the right outcome of the three redundant cores.

This technique applied on image compression of size $904 \mathrm{~K}$. A total overhead of 2187742009 cycles has been recorded, including both the compression and PLR overhead [15]. This technique can only be applied on processing architectures having shared memory protected with hard-ECC schemes.

2) Thread-Level Replication (TLR): This technique is based on the N-version programming [16]. In this scheme, the user function is replicated on three threads, running on different cores in parallel. TMR is used to decide the right core, using majority voter. The abundance of processing resources in Maestro architecture [14] has been harnessed by TLR. The overhead caused by TLR is application dependent [15]. On an empty function the total overhead was 12021270 Cycles. TLR's shared memory requires extra hardware (hard-ECC). 
3) Instructions Level Replication: Decoupled compiler based instruction level fault tolerance (DRIFT) uses the compiler's functionality for error detection. It adds redundant instructions at the compilation process, in addition to inserting check instructions. The goal of DRIFT is to improve the system's performance by reducing the overhead resulted from the error detection, without affecting the error coverage. The overhead is reduced by decoupling the execution of the original code and its replica, and implementing checks [17]. This research was tested using Mediabench II video [18] and SPEC CPU2000 [19]. The average time overhead of this protection technique was $29 \%$. A key issue is that the memory of the processing architecture is assumed to be protected and because this technique only covers the CPU, only allows this technique to be applied to special architectures (memory with hard-ECC).

In the composite datatype protection (CDTP) algorithm, variables are encoded to enable their protections. Memory accessing operations are replaced with new encoded ones. Operations for writing to memory are decoded to check for errors [20]. The cc1 compiler was used to implement the CDTP algorithm automatically as an independent phase of the compilation. The protected code has to be transformed to GIMPLE internal representation [20]. The protection code is added at the initial optimization stage.

The overhead is application dependent and differs from one benchmark to another. The overhead also depends on the protection algorithm used. Using the Hamming code produce $86 \%$ time overhead, extended Golay algorithm 146\%, full iterated coding scheme $116 \%$, and selective iterated coding scheme $117 \%$, which are very large compared to DRIFT. Using encoding and decoding protection schemes is only suitable for the protection of the memory system of the processing architecture; the CPU is still vulnerable to SEEs in this case.

SWIFT-R uses instruction redundancy to detect and correct errors. It adds two replicas of an instruction to the original code, and then calls a TMR voter to decide by majority the correct instruction outcome. This technique does not protect all instructions, especially the memory ones (store, load, alloca, and GetElementPointer) and the branch instructions. Soft errors originating from the SEEs are random in nature, and all instruction types are susceptible to them [21]. Protecting all instruction types will improve the reliability of the processing architecture. This technique adds up to $198 \%$ time overhead of the execution time.

Further techniques include Shoestring [22], fault tolerance software checking [23], error detection by duplicated instructions (EDDI) [24], and software implemented fault tolerance (SWIFT) [25]. The mentioned schemes are implemented by modifying compilers, the LLVM, the GCC, and the OpenIMPACT [26], respectively. The modifications include adding redundant instructions and inserting compare instructions if needed. The mentioned techniques in this section are only for CPU protection, except for EDDI, which is capable of covering both the CPU and memory from the SEEs, but EDDI is architecture dependent and works exclusively on MIPS architectures, and it has no recovery scheme (EDDI detects errors only, and has no recovery).
TABLE I

NOTATION

\begin{tabular}{|c|c|}
\hline Symbol & Quantity \\
\hline$\lambda$ & Failure rate, bits/s \\
\hline$\lambda_{c}$ & Failure rate in cache memory, bits/s \\
\hline$\lambda_{p}$ & Failure rate in $\mathrm{CPU}, \mathrm{bits} / \mathrm{s}$ \\
\hline$W$ & No. of bits per word, bits \\
\hline$r(t)$ & Reliability of one word \\
\hline$R_{r}(t), R_{1}(t), R_{2}(t) \& R_{3}(t)$ & $\begin{array}{l}\text { Reliabilities in the RAM \& different cache } \\
\text { levels }\end{array}$ \\
\hline$R_{\text {rinj }}(t), R_{\text {linj }}(t), R_{2 i n j}(t) \& R_{3 i n j}(t)$ & $\begin{array}{l}\text { Reliabilities in the RAM \& different cache } \\
\text { levels, when errors are injected }\end{array}$ \\
\hline$R_{C P U}(t)$ & The reliability of the CPU \\
\hline$R_{\text {CPUinj }}(t)$ & $\begin{array}{l}\text { The reliability of the CPU when errors are } \\
\text { injected }\end{array}$ \\
\hline$N_{i}$ & Total No. of instructions of type i \\
\hline$H_{l}, M_{l}$ & Hit \& miss rate for L1 (local) \\
\hline $\mathrm{H}_{2}, \mathrm{M}_{2}$ & Hit \& miss rate for L2 (local) \\
\hline$H_{3}, M_{3}$ & Hit \& miss rate for L3 (global) \\
\hline$H_{m}, M_{m}$ & Hit \& miss rate for RAM (global) \\
\hline$H_{2} M_{1}=X_{2}$ & Rate of accessing L2 \\
\hline$H_{3}-\left(H_{1}+M_{1} H_{2}\right)=X_{3}$ & Rate of accessing L3 \\
\hline $1-H_{1}-X_{2}-X_{3}=X_{m}$ & Rate of accessing RAM \\
\hline$f$ & Clock frequency, $\mathrm{Hz}$ \\
\hline$I P S_{0}$ & Instructions per second (single core) \\
\hline$I P C_{N}$ & Instructions per cycle ( $\mathrm{N}$ core) \\
\hline$I P S_{N}=I P C_{N} f$ & Instructions per second ( $\mathrm{N}$ core) \\
\hline$S P_{l}=I P C_{N} f / I P S_{0}$ & Speedup caused by the multicore \\
\hline$M$ & No. of pipeline stages \\
\hline$S P_{2}=N_{i} m /\left(m+N_{i}-1\right)$ & Speedup caused by the pipeline \\
\hline$S_{i}$ & $\begin{array}{l}\text { The error rate from the injection experiment } \\
\text { of instruction type } i \text { (sensitivity ratio) }\end{array}$ \\
\hline$N_{i 0}$ & No. of instructions in the CPU (single core) \\
\hline$N_{c 0}$ & No. of cycles (single core) \\
\hline$N_{1}, N_{2} \& N_{3}$ & No. of cache words used at runtime \\
\hline$S_{i p}$ & $\begin{array}{l}\text { Sensitivity of instructions type i after } \\
\text { protection }\end{array}$ \\
\hline$N_{i p}$ & No. of protected instructions type i \\
\hline$S_{\text {cpup }}, S_{m p}$ & $\begin{array}{l}\text { Sensitivities of CPU \& memory instructions } \\
\text { after protection }\end{array}$ \\
\hline$N_{c p u p}, N_{m p}$ & $\begin{array}{l}\text { No. of CPU \& memory instructions } \\
\text { protected }\end{array}$ \\
\hline$l$ & $\begin{array}{l}\text { Total No. of instruction types of the } \\
\text { benchmark under examination. }\end{array}$ \\
\hline
\end{tabular}

DAFT [27] and SRMT [28] techniques use LLVM, Intel production and ICC 9.0 compilers, respectively, to automate the generation of redundant threads for an application, and then inserts comparisons for their outcome to detect errors. DAFT has better performance compared to SRMT. Both of these techniques are for CPU protection, the memory system is still vulnerable to the SEEs soft errors.

CASTED [29] is implemented at the backend stage of the compilation process; it uses the GCC-4.5.0 [30] compiler framework. This technique includes two passes, one for error detection and another one for recovery. The cache memory is not protected since CASTED only targets CPU protection.

\section{Summary}

The trend in software mitigation against SEUs of radiation effects is to detect errors, and disregard the error recovery part. 


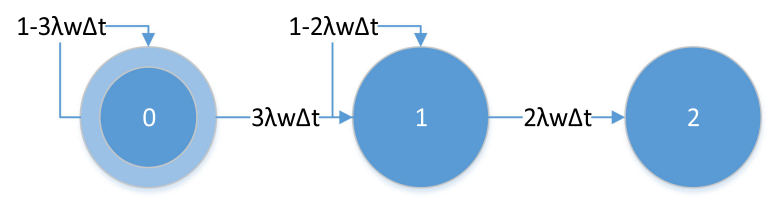

Fig. 1. Markov TMR states.

Another assumption is that the memory system has hard-ECC, which is not affordable in some cases. The addition of software protection techniques will always add extra overhead, depending on the scheme used and the benchmark. Our solution to the SEUs problem is to find an optimal trade between reliability and performance, without ignoring the protection of cache memory system of the processing architecture.

\section{RELIABILITY PREDICTIONS}

In this section, a novel model for the reliability of the protected and unprotected processing architecture was developed, starting from modeling the reliability of the basic components, where the different processor parts, like the CPU, caches, and RAM, have been modeled, and using combinational logic, the reliability of the whole system has been modeled. This section is a preparation for the next one, where the reliability of the predictions of the protected architecture, will be compared with the reliability obtained from the fault injection experiment of the protected code.

In the predictions for the reliability of the unprotected code, the sensitivity of every instruction type to the fault injection caused by our software to simulate SEE will be included. The sensitivity of an instruction type is the number of errors divided by the total number of injections.

Redundancy has been an efficient way of protecting memory systems. A nonredundant memory system fails if a fault occurs in one of its words. Assuming Poisson process, and the statistical independence among failures, the reliability of the memory system is the product of the reliabilities of all its $N$ words

$$
R(t)=e^{(-\lambda \mathrm{WN} t)} .
$$

The failure rate for each word in the TMR system is $\lambda$ and $\mathrm{W}$; the Markov chain is given in Fig. 1 [31].

State 0 represents the state where all the TMR bits in the words are correct. State 1 represents the case where a fault has occurred in any of the three replicated words. State 2 represents the state where more than one word has an error.

The following set of differential equations represents the dynamics of the probability system:

$$
\begin{aligned}
& \frac{d P_{0}(t)}{d t}=-3 \lambda \mathrm{W} P_{0}(\mathrm{t}) \\
& \frac{d P_{1}(t)}{d t}=3 \lambda \mathrm{W} P_{0}(\mathrm{t})-2 \lambda \mathrm{W} P_{1}(\mathrm{t}) \\
& \frac{d P_{2}(t)}{d t}=2 \lambda \mathrm{W} P_{1}(\mathrm{t}) .
\end{aligned}
$$

Using Laplace transforms, and assuming that the system starts without faults at time $t=0$, meaning, $P_{0}(0)=1$, and

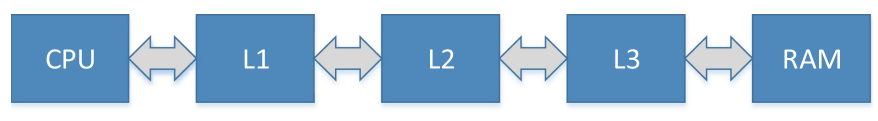

Fig. 2. Memory modules connection in software perspective.

$$
\begin{aligned}
& P_{1}(0)=P_{2}(0)=0 . \text { We obtain } \\
& P_{0}(t)=e^{-3 \lambda \mathrm{W} t} \\
& P_{1}(t)=3 e^{-2 \lambda \mathrm{W} t}-3 e^{-3 \lambda \mathrm{W} t} \\
& P_{2}(t)=1-3 e^{-2 \lambda \mathrm{W} t}+2 e^{-3 \lambda \mathrm{W} t} .
\end{aligned}
$$

The reliability using TMR is

$$
r(t)=3 e^{-2 \lambda \mathrm{W} t}-2 e^{-3 \lambda \mathrm{W} t} .
$$

The final expression in (8) represents the reliability of a single word of W bits, when protected with TMR.

\section{A. Reliability Prediction for $N$ Word Memory With TMR}

The software TMR is a serial combination of $N$ TMRs, if two errors occur on the same TMR causing it to fail, the whole memory system will fail. The expression for reliability for TMR without repair is

$$
R(t)=\left(3 e^{(-2 \lambda \mathrm{W} t)}-2 e^{(-3 \lambda \mathrm{W} t)}\right) \cdot{ }^{N}
$$

\section{B. Reliability Prediction of RAM, Caches, and CPU Without Protection}

In this section, the reliability of the whole processing architecture was deduced. The reliabilities of the RAM, caches, and CPU were combined, knowing that the components are serially connected in Fig. 2, and taking into consideration that the error rate $\lambda$ changes depending on the component's cross-section area.

The reliabilities for each component are given by the following expressions:

$$
\begin{aligned}
\mathrm{R}_{1}(\mathrm{t}) & =\mathrm{e}^{\left(-\mathrm{WH}_{1} \lambda_{c} \mathrm{t} \sum_{i=0}^{i=l} S_{i} N_{i}\right)} \\
\mathrm{R}_{2}(\mathrm{t}) & =\mathrm{e}^{\left(-\mathrm{WX}_{2} \lambda_{c} \mathrm{t} \sum_{i=0}^{i=l} \mathrm{~S}_{i} N_{i}\right)} \\
\mathrm{R}_{3}(\mathrm{t}) & =\mathrm{e}^{\left(-\mathrm{WX}_{3} \lambda_{c} \mathrm{t} \sum_{i=0}^{i=l} \mathrm{~S}_{i} N_{i}\right)} \\
\mathrm{R}_{r}(\mathrm{t}) & =\mathrm{e}^{\left(-\mathrm{WX}_{\mathrm{r}} \lambda_{r} \mathrm{t} \sum_{i=0}^{\mathrm{i}=1} \mathrm{~S}_{i} N_{i}\right)} \\
\mathrm{R}_{\mathrm{CPU}}(\mathrm{t}) & \left.=\mathrm{e}^{\left(-\mathrm{W} \frac{\sum_{i=0}^{i=l} S_{i} N_{i}}{\mathrm{SP}_{1} \mathrm{SP}_{2}} \lambda_{p} \mathrm{t}\right.}\right) .
\end{aligned}
$$

In addition to error rates $\lambda$ 's, we also introduce a new variable: The instruction sensitivity $S_{\mathrm{i}}$, which changes from one instruction type to another for each benchmark. The sensitivity of an instruction type is the total number of errors caused by injection divided by total the number of injections. For small benchmarks, the number of instruction types is equal to the number of injections, but may differ in case of large benchmarks where sampling for a specific confidence level must be reached for valid results. 
In order to determine the sensitivity of the instruction types of a certain benchmark, all the instructions of this type must be injected (can also inject a significant sample size, in case the benchmark has a large number of instructions).

The reliability in the caches and the RAM is effected by the access rates, where the first level of cache has the highest rate, and the rate drops with every subsequent cache level, until the RAM with the slowest access rate.

In the CPU, the reliability is effected by two factors, the speed up caused by the multicore $\mathrm{SP}_{1}$ and the speed up caused by the number of pipeline stages $\mathrm{SP}_{2}$. We are assuming that the speedup improves the reliability, since it improves the time of execution, reducing the probability of catching a SEE.

The reliability $R(\mathrm{t})$ expression of the whole system is given by the following equation:

$$
R(t)=R_{r}(t) R_{3}(t) R_{2}(t) R_{1}(t) R_{\mathrm{CPU}}(t) .
$$

\section{TMR Protected}

The reliability predictions of cache levels RAM and CPU when the ACEDR protection is applied are given by the following expressions:

$$
\begin{aligned}
R_{1}(\mathrm{t})= & \prod_{i=0}^{i=l}\left(3 e^{-2 \mathrm{~W} S_{i} \lambda_{c} t}-2 e^{-3 \mathrm{~W} S_{i} \lambda_{c} t}\right)^{H_{1} N_{i}} \\
R_{2}(t)= & \prod_{i=0}^{i=1}\left(3 e^{\left(-2 \mathrm{~W} S_{i} \lambda_{c} t\right)}-2 e^{\left(-3 \mathrm{~W} S_{i} \lambda_{c} t\right)}\right)^{X_{2} N_{i}} \\
R_{3}(t)= & \prod_{i=0}^{i=l}\left(3 e^{\left(-2 \mathrm{~W} S_{i} \lambda_{c} t\right)}-2 e^{\left(-3 \mathrm{~W} S_{i} \lambda_{c} t\right)}\right)^{X_{3} N_{i}} \\
R_{r}(t)= & \prod_{i=0}^{i=1}\left(3 e^{\left(-2 \mathrm{~W} S_{i} \lambda_{r} t\right)}-2 e^{\left(-3 \mathrm{~W} S_{i} \lambda_{r} t\right)}\right)^{X_{r} N_{i}} \\
R_{\mathrm{CPU}}(t)= & \prod_{i=0}^{i=1}\left(3 e^{\left(-2 \mathrm{~W} S_{i} \lambda_{p} t\right)}\right. \\
& \left.-2 e^{\left(-3 \mathrm{~W} S_{i} \lambda_{p} t\right)}\right)^{S_{i} \frac{N_{i}}{\mathrm{SP}_{1} \mathrm{SP}_{2}}} .
\end{aligned}
$$

The reliability of the whole protected system combined is

$$
R(t)=R_{r}(t) R_{3}(t) R_{2}(t) R_{1}(t) R_{\mathrm{CPU}}(t) .
$$

\section{Reliability Equations Obtained From the Injection Experiments}

In order to compare the theoretical predictions of the reliability of the protected code in Section III-C, we modeled a new reliability expression of the whole processing architecture chain after it is protected with ACEDR for the fault injection experiment. The difference between reliability of the protected and the unprotected codes is the sensitivity of instructions to error that will drop after the protection is added, leading to the following equations representing the reliability of every processing component (different levels of caches, RAM, and the CPU):

$$
R_{1 i n j}(t)=\mathrm{e}^{\left(-\mathrm{WH}_{1} \lambda t \sum_{i=0}^{i=l} S_{i p} N_{i p}\right)}
$$

$$
\begin{aligned}
R_{2 i n j}(t) & =\mathrm{e}^{\left(-\mathrm{WX}_{2} \lambda t \sum_{i=0}^{i=l} S_{i p} N_{i p}\right)} \\
R_{3 i n j}(t) & =\mathrm{e}^{\left(-\mathrm{WX}_{3} \lambda t \sum_{i=0}^{i=l} S_{i p} N_{i p}\right)} \\
R_{\text {rinj }}(t) & =\mathrm{e}^{\left(-\mathrm{WX}_{r} \lambda t \sum_{i=0}^{i=l} S_{i p} N_{i p}\right)} \\
R_{\text {CPUinj }}(t) & =\mathrm{e}^{\left(-\mathrm{W} \frac{\sum_{i=0}^{i=l} S_{i p} N_{i p}}{\mathrm{SP}_{1} \mathrm{SP}_{2}} \lambda t\right)} .
\end{aligned}
$$

The reliability of the whole system is given by

$$
\begin{aligned}
R_{\text {inj }}(t)= & R_{1 i n j}(t) \cdot R_{2 i n j}(t) \cdot R_{3 i n j}(t) \cdot R_{\text {rinj }}(t) \\
& \cdot R_{\text {CPUinj }}(t) .
\end{aligned}
$$

\section{E. Application of the Reliability Predictions (Relevance of the Prediction Model to the Rest of the Paper)}

The main objective of adding this section is to identify the precision of the prediction model, by comparing (27) to (21). This section is combining the results obtained from the theoretical model to the results obtained from the experimental injection in Section $\mathrm{V}$, where the injection experiment is necessary to obtain the different instructions sensitivities used in the prediction models.

When the injection experiments of all instruction types were performed in our previous work [32], an observation has been made on the generic categories of instruction types that cause significant changes in the error rates, these categories are the CPU and Memory instructions. In this section, we will apply the reliability equations shown previously on the CPU and Memory instructions generic categories.

This means that we have two types of sensitivities, $S_{\text {cpu }}$ representing the sensitivity of the CPU instructions and $S_{m}$ representing the sensitivity of the memory instructions.

The resulting equations for the reliability prediction for no protection are obtained using (15)

$$
e^{-t \mathrm{~W}\left(\lambda_{r} H_{r}+\left(\frac{\lambda_{p}}{\operatorname{SP} 1 \mathrm{SP} 2}\right)+\lambda_{c}\left(H_{1}+X_{2}+X_{3}\right)\right)\left(S_{\mathrm{cpu}} N_{\mathrm{cpu}}+S_{m} N_{m}\right)} .
$$

The equation of the reliability prediction after adding protection is obtained using (16)-(20)

$$
\begin{aligned}
R_{1}( & t) \\
= & \left(3 e^{\left(-2 \mathrm{~W} S_{\mathrm{cpu}} \lambda_{c} t\right)}\right. \\
& \left.-2 e^{\left(-3 \mathrm{~W} S_{\mathrm{cpu}} \lambda_{c} \cdot t\right)}\right)^{H_{1} \cdot N_{\mathrm{cpu}}}\left(3 e^{\left(-2 \mathrm{~W} S_{m} \lambda_{c} t\right)}\right. \\
& \left.-2 e^{\left(-3 \mathrm{~W} S_{m} \lambda_{c} t\right)}\right)^{H_{1} N_{m}} \\
R_{2}( & t) \\
= & \left(3 e^{\left(-2 \mathrm{~W} S_{\mathrm{cpu}} \lambda_{c} t\right)}\right. \\
& \left.-2 e^{\left(-3 \mathrm{~W} S_{\mathrm{cpu}} \lambda_{c} t\right)}\right) X_{2} N_{\mathrm{cpu}}\left(3 e^{\left(-2 \mathrm{~W} S_{m} \lambda_{c} t\right)}\right. \\
& \left.-2 e^{\left(-3 \mathrm{~W} S_{m} \lambda_{c} t\right)}\right)^{X_{2} N_{m}} \\
R_{3}( & t) \\
= & \left(3 e^{\left(-2 \mathrm{~W} S_{\mathrm{cpu}} \lambda_{c} t\right)}\right. \\
& \left.-2 e^{\left(-3 \mathrm{~W} S_{\mathrm{cpu}} \lambda_{c} t\right)}\right)^{X_{3} N_{\mathrm{cpu}}}\left(3 e^{\left(-2 \mathrm{~W} S_{m} \lambda_{c} t\right)}\right. \\
& \left.-2 e^{\left(-3 \mathrm{~W} S_{m} \lambda_{c} t\right)}\right)^{X_{3} N_{m}}
\end{aligned}
$$




$$
\begin{aligned}
R_{r}( & t) \\
= & \left(3 e^{\left(-2 \mathrm{~W} S_{\mathrm{cpu}} \lambda_{r} t\right)}\right. \\
& \left.-2 e^{\left(-3 \mathrm{~W} S_{\mathrm{cpu}} \lambda_{r} t\right)}\right)^{X_{r} N_{\mathrm{cpu}}}\left(3 e^{\left(-2 \mathrm{~W} S_{m} \lambda_{r} t\right)}\right. \\
& \left.-2 e^{\left(-3 \mathrm{~W} S_{m} \lambda_{r} t\right)}\right)^{X_{r} N_{m}} \\
R_{\mathrm{CPU}} & (t) \\
= & \left(3 e^{\left(-2 \mathrm{~W} S_{\mathrm{cpu}} \lambda_{p} t\right)}\right. \\
& \left.-2 e^{\left(-3 \mathrm{~W} S_{\mathrm{cpu}} \lambda_{p} t\right)}\right) \frac{N_{\mathrm{CPU}}}{\mathrm{SP}_{1} \mathrm{SP}_{2}}\left(3 e^{\left(-2 \mathrm{~W} S_{m} \lambda_{p} t\right)}\right. \\
& \left.-2 e^{\left(-3 \mathrm{~W} S_{m} \lambda_{p} t\right)}\right)^{\frac{N_{m}}{\mathrm{SP}_{1} \mathrm{SP}_{2}}} .
\end{aligned}
$$

The reliability of the whole system is given by (21).

When taking the CPU and memory instruction types, the reliability of the protected processor from the injection experiment is obtained using (27). The reliability of the whole architecture is

$$
\begin{aligned}
R_{i n j}(t)= & e^{-} t W\left(\left(\lambda_{r} H_{\mathrm{r}}+\left(\frac{1}{\mathrm{SP}_{1} \mathrm{SP}_{2}} \lambda_{\mathrm{p}}\right)\right.\right. \\
& \left.+\lambda_{\mathrm{c}}\left(H_{1}+X_{2}+X_{3}\right)\right) \\
& \left.\left(S_{\text {cpup }} N_{\text {cpup }}+S_{m p} N_{m p}\right)\right) .
\end{aligned}
$$

\section{F. Summary}

In this section, we have shown the reliability models of the protected and unprotected codes, using the software protection techniques that have been developed. Using combinational logic, and including multiple parameters in the model, aiming to have the first in-depth reliability prediction that can be valid for the COTS processing architectures.

At the start, the reliability of the unprotected processor was shown in (15), followed by the reliability of the protected system (21) and at last, (34) for the reliability obtained from the injection experiment of the protected code.

In order to check the precision of our prediction model, the reliability obtained from (21) and (34) will be compared; this will show how close the theoretical and experimental reliabilities are to each other. In this model, we consider (34) as the ground truth. Another comparison between (21) and (34) will show how much reliability was added using the software protection techniques.

At this point, the prediction models have been obtained. In order to perform the different comparisons, we will need to obtain the different sensitivities of the different instruction types, and by sensitivity, it means the error rate of every instruction type. In order to obtain the sensitivity of the instructions, the injection experiments must be performed on both the protected and the unprotected codes.

\section{Automatic Compiler ERror Detection AND RECOVERY}

Based on our previous works [10] and [32], a decision has been made to use TMR for error detection and recovery, with its ability to detect and recover without adding large overhead, compared to the implementation of the Hamming and BCH codes. Nevertheless, our method provides a way to implement any error detection and recovery technique at the instruction level.

LLVM compiler framework is the backbone of this paper. The implementation is achieved at the optimizer stage of the compilation process by creating LLVM passes to enable the automatic addition of protection code to any benchmark that can be compiled with LLVM. The code we intend to protect must be in its IR, which can be achieved by using the "clang" compiler front end. One major advantage of using our software protection technique is that the user does not have to write a single line of protection code, all they need to do is compile the unprotected code using our passes and the code will be protected.

As discussed, the protection code includes analysis and transformation passes. The analysis pass will provide us with statistic information about the code; it also goes through all instructions and determines their types. The transformation pass is the part that adds the protection code; it uses the information provided by the analysis to make the decision of the protection to be added [10].

In order to choose the best protection codes in terms of performance, we conducted a study of the overhead generated by applying different protection schemes [10]. Hamming code has been implemented first in the study of ACEDR. Hamming code is able to detect two errors and recover single one. The $\mathrm{BCH}$ protection scheme has also been studied, with its ability to detect and recover multiple error bits.

The use of both the Hamming and $\mathrm{BCH}$ codes has shown a large overhead, so we decided to exclude these techniques from our automatic compiler implementation. Another constraint is that all ECC codes, including the Hamming and $\mathrm{BCH}$, are more suitable for memory systems protection, where the writing is done with the encoder of the ECC, and the reading is done with the decoder of the ECC in order to detect and recover errors. CPU instructions redundancy schemes, like the NMR techniques, are most suitable to detect and recover the errors.

The TMR implementation using automatic compiler passes has shown the best performance with its low overhead [10]. This technique has also shown the ability to detect and recover any single bit errors. TMR is also able to detect and recover multiple error bits, with the condition that all the errors occur on the same word of the TMR'd words, or the two errors occur on two different words of two different TMR'd words. The TMR's limitation is in case of the occurrence of two or more errors in two different words of the same TMR'd words. In this case, TMR will still be able to detect the occurrence of an error, but it cannot recover, since it does not know which word is correct.

\section{A. ACEDR Principles}

In this section, we explain the concept of protection using LLVM passes that will add protection code. We divide instructions to two main categories Memory (R/W operations) and CPU (Arithmetic and logic operations) in order to show that the protection of both types is important. SEEs occur randomly, meaning that all instruction types are vulnerable to the radiation effects. In the literature, memory instructions are typically 


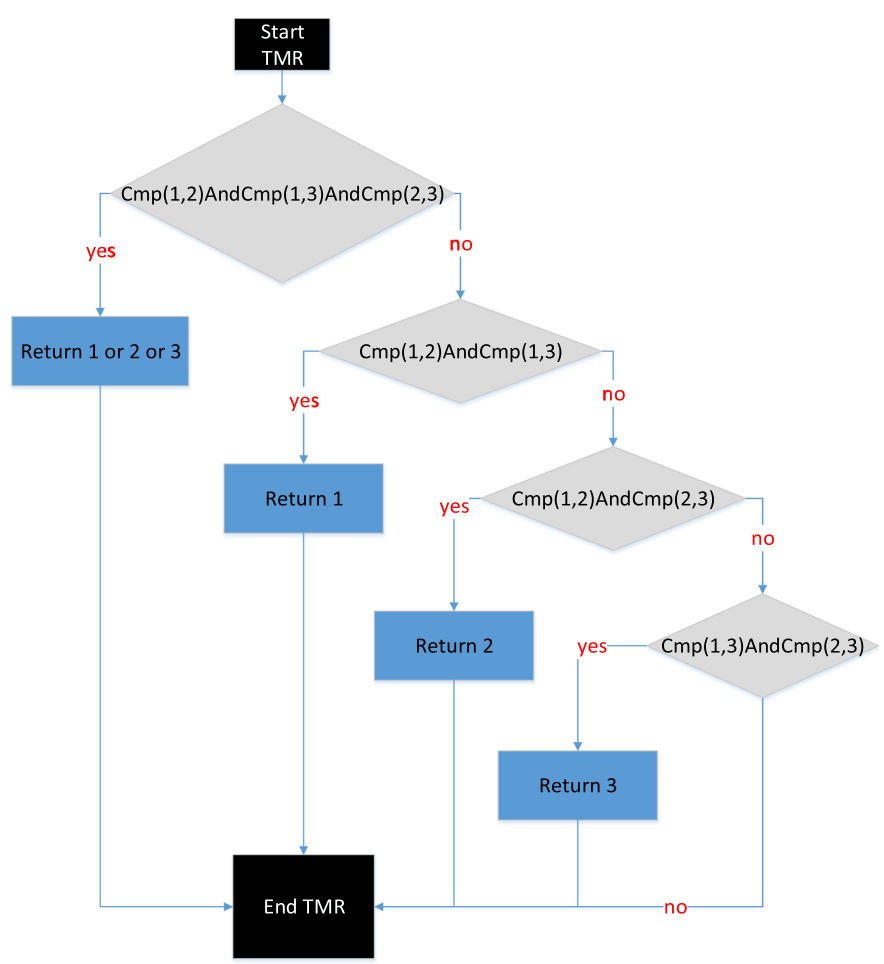

Fig. 3. Software TMR algorithm.

protected with external hardware, such as hard-ECC circuits; that is why most software protection techniques do not provide schemes for cache memory protection. This paper does not make any presumptions on the chosen architecture's memory system and considers it to be unprotected. As our research studies SEEs on both the cache memory and CPU instructions, both require injection. Unlike the literature, where only the CPU instructions have been injected, we inject all instructions to understand their consequences. Adding the protection at the optimizer of the LLVM compilation process will allow this research to target multiple processing architectures, and multiple high level programming languages. After the code is transformed to its IR, the passes run on it, and add the protection code automatically. The addition of instructions can be achieved using the using clone () [33], or by building new instructions using the IRBuilder [34]. The addition of the protection functions could be achieved directly by embedding them inside the pass or using the linker to link the protection functions with the code to be protected. The second method allows the user to gain more time in the development of the protection code. The protection function is a TMR; it works with the majority vote as shown in Fig. 3.

\section{B. Adding ACEDR Instructions in IR}

Our previous work [10] has been extended to include multiple datatypes (i32, i32 ${ }^{\star}, \mathrm{i} 1, \mathrm{i} 8, \mathrm{i} 8^{\star}$, i64, float and double, float and double pointers). This extension will allow our paper to have high coverage compared to the state of the art. ACEDR-TMR will add two redundant instructions to the original one, and then calls a voter function in order to decide the correct outcome

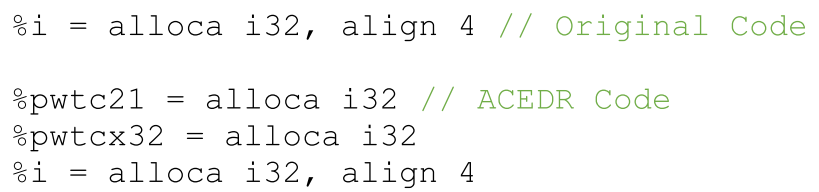

Fig. 4. Protecting the "alloca" memory instruction.

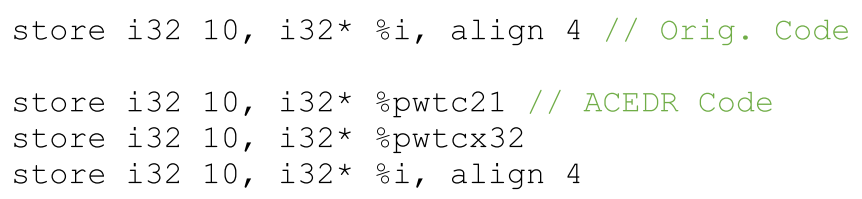

Fig. 5. Protecting the "store" memory instruction.

among the three instructions, the replicated and the original. In this paper, we found that memory instructions (alloca, load, store, and GetElementpointer) and CPU instructions (Arithmetic and logic operators, etc.) both require protection, because we do not assume that the memory system is protected with any type of hard-ECC protection techniques, allowing us to extend our implementation to more processors.

1) Memory Instructions Protection: Memory instructions occupy a large portion of the code, unless there is a hardware dedicated especially for error detection and recovery, like the use of hard-ECC. Then the instructions responsible for R/W from/to memory must be protected, in order to make the processing architecture more resilient.

There is a dependency between memory instruction types, where read and write operations are using the locations that have been created with alloca instructions. Same applies to Getelemnptr (GEP) instructions, where newly created GEP instruction point to previously created allocation. Our software protection scheme detects this dependency relation and adds the appropriate protection code accordingly. At first the newly created allocations are detected, this could be achieved using the analysis pass, providing the number and the types of the alloca. After the alloca type is recognized, the transformation pass will add the protection code. In this case, the transformation pass will create new redundant allocations and each time a read or a write to the original allocations is detected, the same operations will be replicated on the redundant allocations. The alloca instruction replication is shown in Fig. 4. In the following code, \%i is the original alloca instruction and \%pwtc21 and \%pwtcx 32 are the redundant ones we created.

The analysis pass will detect writes to the original allocations (store instructions), which stores a value in an address specified by the allocation. The transformation pass will know the types of the original store instructions and their number, and adds the protection code accordingly. In this case, new stores will be created storing same value of the original one to the newly created allocations previously, Fig. 5. The instruction store $i 3210, i 32^{\star} \% i$ is the original store, store $i 3210$, i32* $\%$ pwtc21, and store $i 3210$, i32* opwtcx32 are the newly created stores. 


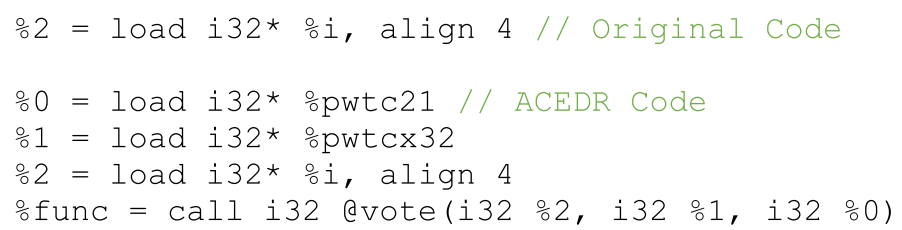

Fig. 6. Protecting the "load" memory instruction.

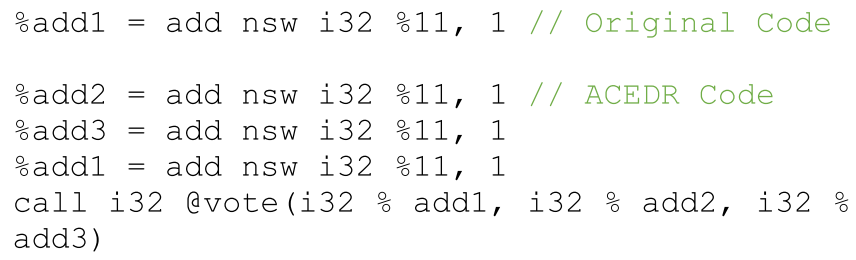

Fig. 7. Protecting the "add" CPU instruction.

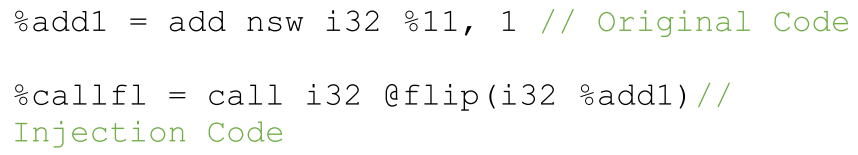

Fig. 8. Injection of CPU instruction.

Every time a load or (read) instruction is detected from a memory location (the original read is $\% 2=10 a d i 32^{\star}$ \% $i$, align 4), redundant reads are added (the redundant reads are $\% 0=1$ oad $i 32^{\star} \%$ pwtc 21 and $\% 1=$ load $132^{\star} \%$ pwtcx 32$)$, and the outcome is compared using a voter $\%$ func $=$ call i32 @vote(i32 \%2, i32 \%1, i32 $\% 0$ ), resulting that the correct memory location only will be the one with the final read Fig. 6 .

2) ALU Instructions Protection: The analysis pass will detect this type of instruction and classify them depending on their data. The transformation pass will know the type of instruction and replicates them, and call a voter function to decide the right outcome at runtime; see Fig. 7.

3) CPU and Memory Protection: At last, the protection of both instruction types will be combined, in order to assess the highest resilience for the processing architecture. This can be achieved with a single transformation pass, two separate ones, or each pass dedicated to protect a certain instructions type.

This combination ensures that memory instructions are protected, meaning that they can be used by the CPU safely. The ALU instructions will also be protected as well by redundancy a voting function checker.

\section{ERROR INJECTION}

We have developed a new fault injection tool based on LLVM compiler in order to validate and measure our software protection method's ability to detect and recover. The process of injection will be applied on the unprotected code, as well as the protected code. By quantifying the number of injections and produced errors, the error coverage can be deduced. For large benchmarks, we inject to produce a 0.95 confidence level.

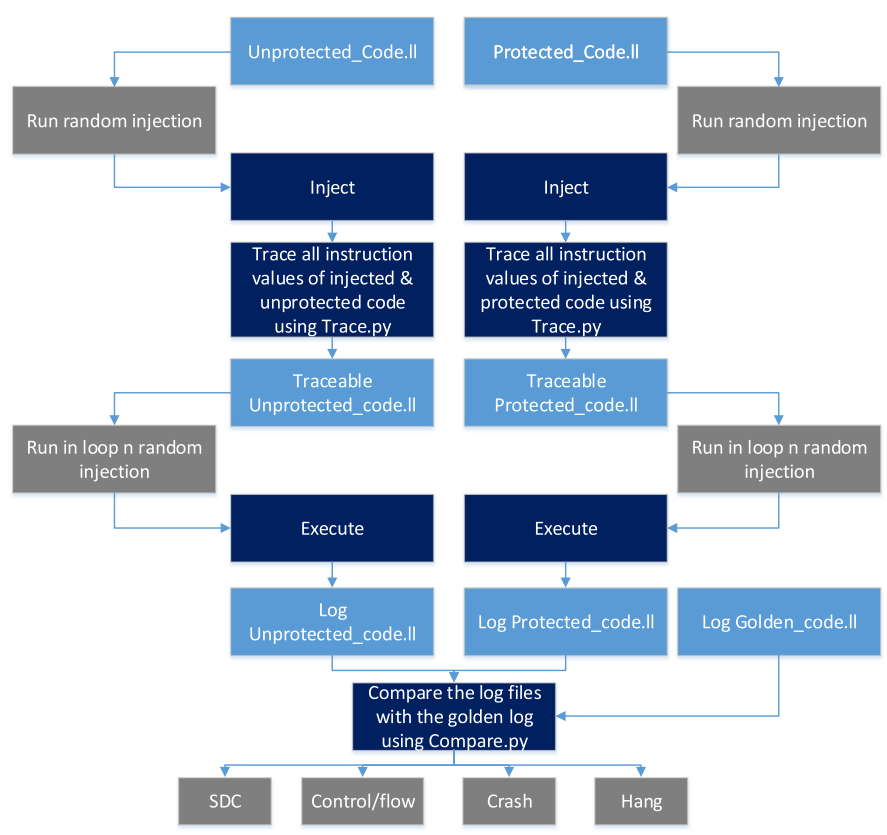

Fig. 9. Injecting, tracing, and comparison of the protected and unprotected code.

store i32 \%add, i32* $\% \times 3$, align 4// Original code

оcall5 = call i32 eflip(i32* \%x3)// Injection Code

store i32 \%call5, i32* $\frac{1}{2} 3$

Fig. 10. Injection of store memory instruction.

The error injector's principle of work is shown in Fig. 9. The left side shows the injection and the tracing of the protected code. Tracing is the process of logging the outcome of all instructions into files. The logged files of the injection experiment will be compared with the golden files (files containing the outcome of every instruction without injection). The code must be in its IR to be traced. Using a python script, tracing instructions are added to the code that logs the outcome of every instruction.

On the right side of Fig. 9, the injection and tracing of the protected code is achieved. This code will be injected after the protection code has been implemented on it, using our LLVM protection passes. After that, the protected code will be injected randomly by calling a function to randomly flip an instruction to emulate a single event error and produce one of the error types (SDC, Control, Hang, or Crash). This depends on the nature of the instruction and how it propagates through the code. Finally, a comparison is done between the golden files and the outcomes of injecting unprotected and protected codes. Comparing the protected injected code log files with the golden ones will show the number and types of errors that occurred, allowing to determine the coverage added by our software protection code. The information provided by the analysis pass will be used by the fault injector in order to make decisions of the injection required to every instruction type. The code will be injected in its IR, where 
every type has its injecting function, including (i32, i32*, i1, i8, i8 $8^{\star}$, i64, float and double, float and double pointers).

Limitations of our fault injector are as follows.

1) We cannot inject void types.

2) Branches are void by nature and, in order to inject them, the decision instruction (ex "cmp") must be injected.

3) Return instructions are void. In order to inject them, the "load" instruction before them must be injected.

\section{A. Injection Experiments of Different Instruction Types}

1) Injecting the CPU Instructions: The following code snippet shows how the injection of the CPU instructions is performed.

Once the instruction has been injected using the $\mathrm{f} I \mathrm{ip}$ function responsible for flipping randomly one if its bits, the following function will be called inside the LLVM injection pass: replaceAllUsesWith (callfl). This function is responsible for replacing the uses of the previous instruction with the newly injected one.

2) Injecting Memory Instructions: The process of injecting the load instructions is similar to the injection of the CPU instructions; the only difference is when injecting the store instruction, which is depicted in the following snippet.

Instead of calling the method responsible for replacing the uses of the store instructions, it is sufficient to add a new store instruction, to store the injected value in the original memory address, where the correct value was supposed to be stored.

The flip function will take a value or pointer to the value, then it will randomly flip one of its 32 bit (in case an int 32 is flipped), then returns the flipped value, or a pointer to the flipped value (if a pointer is passed to it).

In order to detect the different types of error that have occurred, a python script will compare the two log files, of the golden outputs and the injected logs. SDC errors are detected if the output files have same length, but different outcomes. Hangs and Control errors are detected when the injected log files are longer than the golden files. Crash errors are known, when the size of the output injected file is 0 ; see Algorithm 1.

\section{B. Injecting Unprotected Code}

In order to evaluate our method's error detection and recovery ability, we have implemented it on nine known and common benchmarks: Fib, Qsort, SolveCubic, Rad2Deg, Deg2Rad, UQsort, fast Fourier transformations (FFT), Matrix multiplication (MM) and Suzan from MediaBench [35]. SolveCubic, Rad2Deg, Deg2Rad, UQsort were all combined to a single benchmark we called Math benchmark.

The unprotected code is highly vulnerable to error injection, since it has no protection scheme. All benchmarks, except for Susan, have shown more than $50 \%$ error rates. Hangs only occur in the Susan benchmark. All of the benchmarks suffered SDC, control errors, and crashes, except for Fibo benchmark where only SDC and control errors occurred; see Fig. 11.

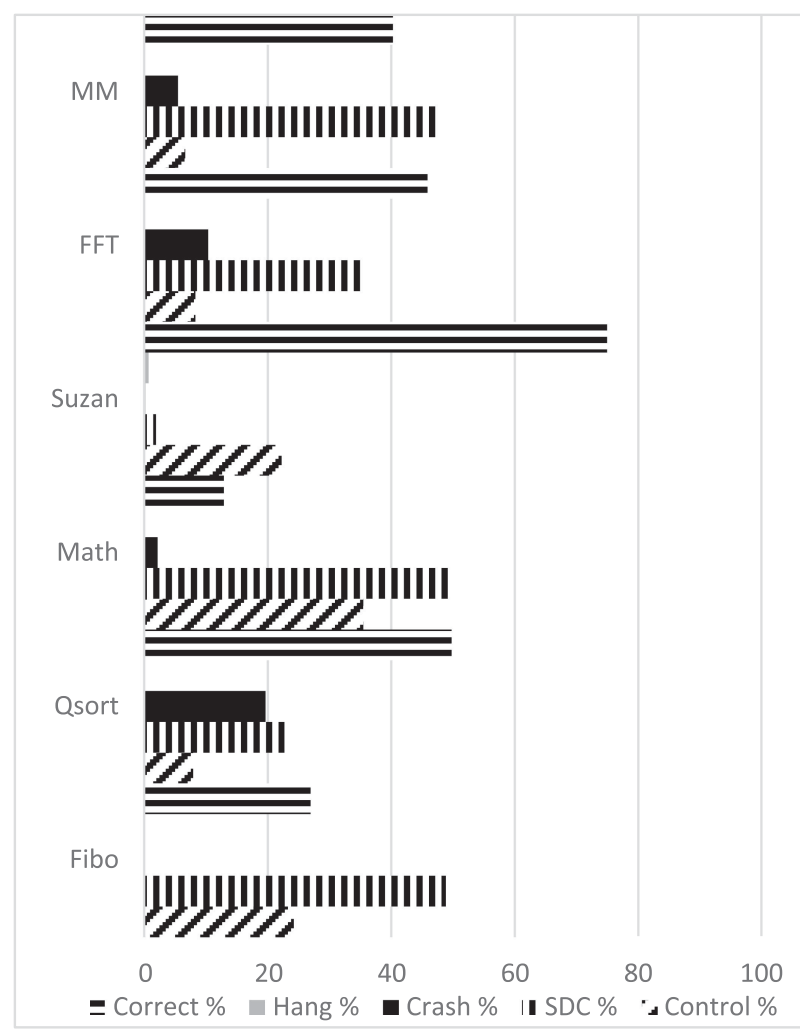

Fig. 11. No protection applied.

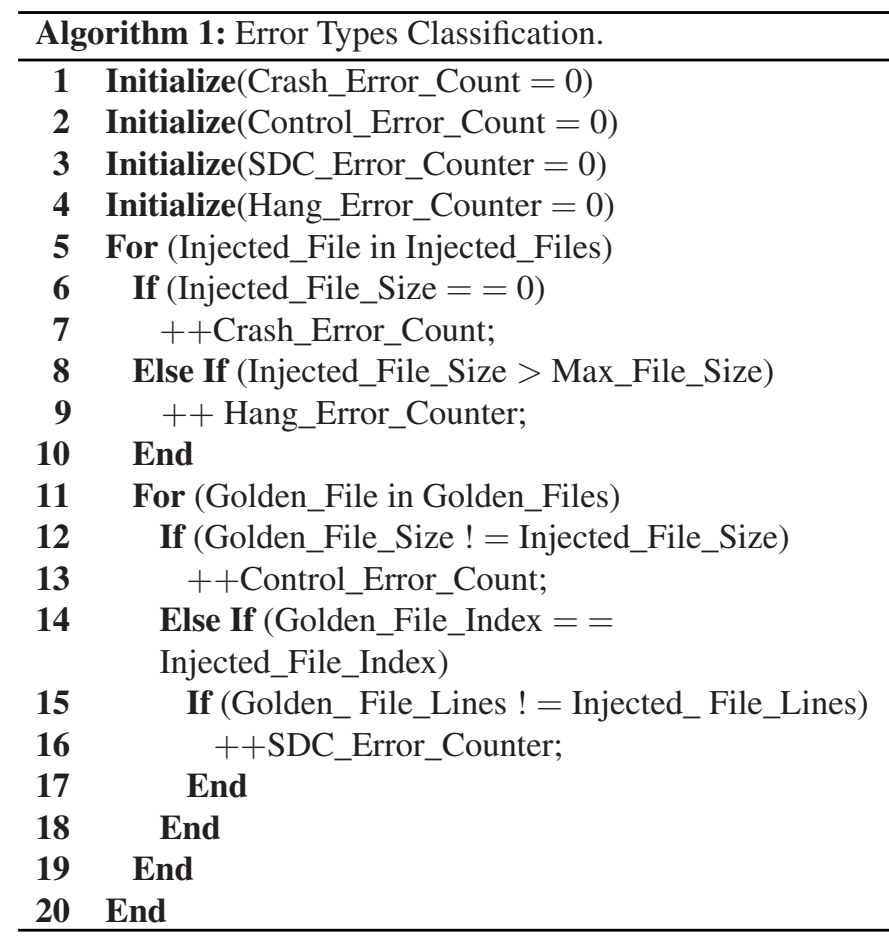

\section{Protecting CPU Instruction Types}

Unlike most of the literature that injects only CPU instructions, we decided to take a more holistic and realistic approach by injecting all instruction types to assess whether our protection technique could reduce the original error rates obtained from 


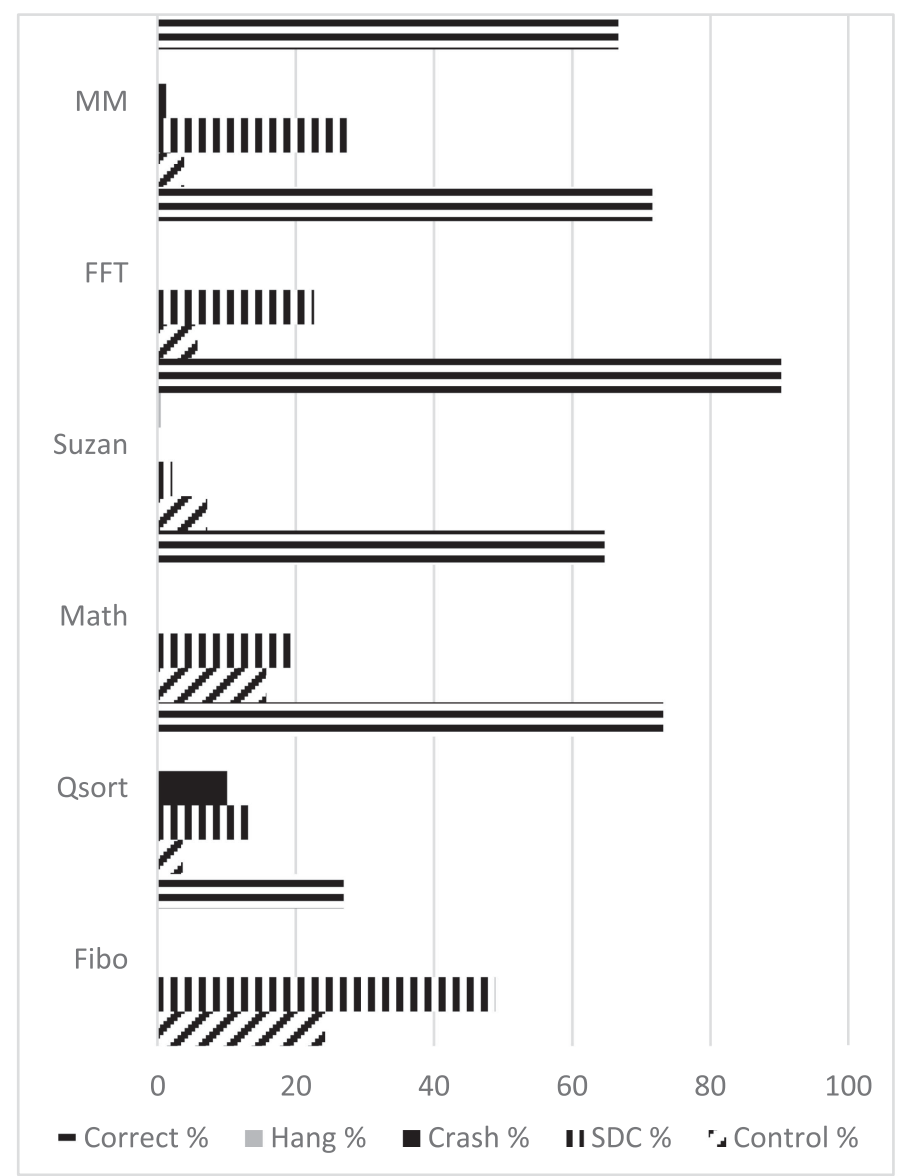

Fig. 12. Protect CPU instructions (binary operations, arithmetic and logic instructions).

the first experiment. In our second injection experiment, only the CPU instructions (and not cache memory) have been protected.

Fig. 12 shows that the error rate has been reduced slightly in most of the benchmarks. In the Fibo benchmark, protecting only CPU instructions has not reduced the error rate and stayed similar to the unprotected experiment. The error rates have been dropped to $9.78 \%, 26.85 \%, 33.36 \%, 28.41 \%$, and $33.35 \%$ in $\mathrm{Su}-$ san, Qsort, Math, FFT, and MM benchmarks, respectively. This shows that protecting only the CPU instructions is not enough to guarantee good error coverage. This high error rate is due to the nature of benchmarks where the CPU instructions occupy a small part of the code.

\section{Protecting Memory Instruction Types}

In this third experiment, only memory instructions have been protected, including the read and write operations from/to cache memory. In the literature, this type of instruction has been ignored, because of the assumption of using hard-ECC. We do not assume having any special hardware architecture, allowing us to extend our paper to multiple processing architectures. In this experiment we injected all the instruction types and checked the error coverage. The error rate has been reduced by just protecting the memory instructions, compared to the second experiment

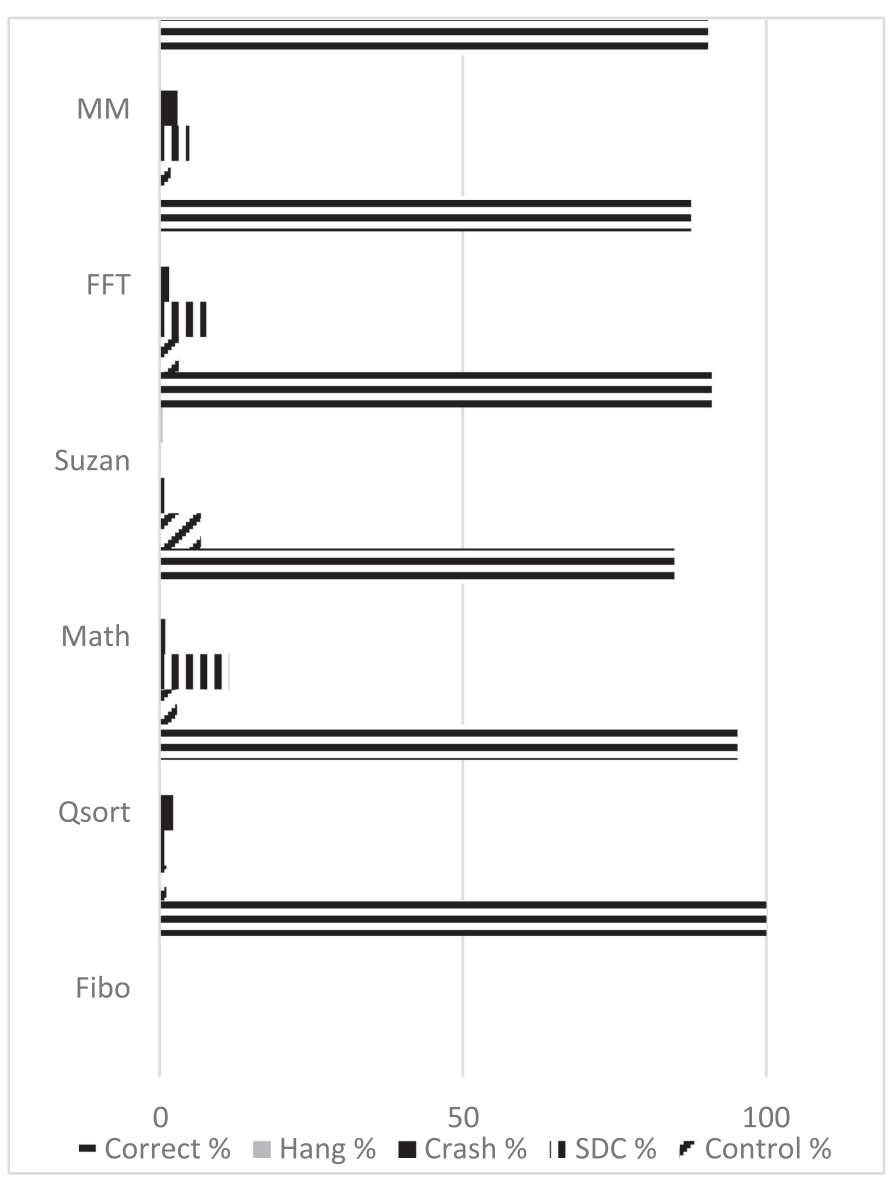

Fig. 13. Protect memory instructions.

where only the CPU instructions have been protected. The error rates have been dropped to $0 \%, 4.77 \%, 9.02 \%, 15.18 \%, 12.40 \%$, and $9.62 \%$ in Fibo, Qsort, Susan, Math, FFT, and MM benchmarks, respectively; see Fig. 13. This improvement has been made due to the high portion occupied by memory instructions in the benchmarks, meaning this type of instruction requires protection.

\section{E. Protecting All Instruction Types}

Injecting the protected code and comparing it to the injected unprotected code will quantify the reliability provided by our protection technique to the benchmarks. In the fourth experiment all instruction types have been protected. This means the combination of protection of both the CPU and memory instructions.

This has dramatically improved the coverage where in some benchmarks the error rate has been reduced to $0 \%$. In the Math benchmark, including (SolveCubic, Rad2Deg, Deg2Rad, and UQsort), FFT and MM benchmarks, the total error has been dropped to $3.97 \%, 1.23 \%$, and $0.71 \%$, respectively. For the Susan benchmark where random errors have been injected, we noticed that the error rate has been dropped to $0.83 \%$; see Fig. 14 . 


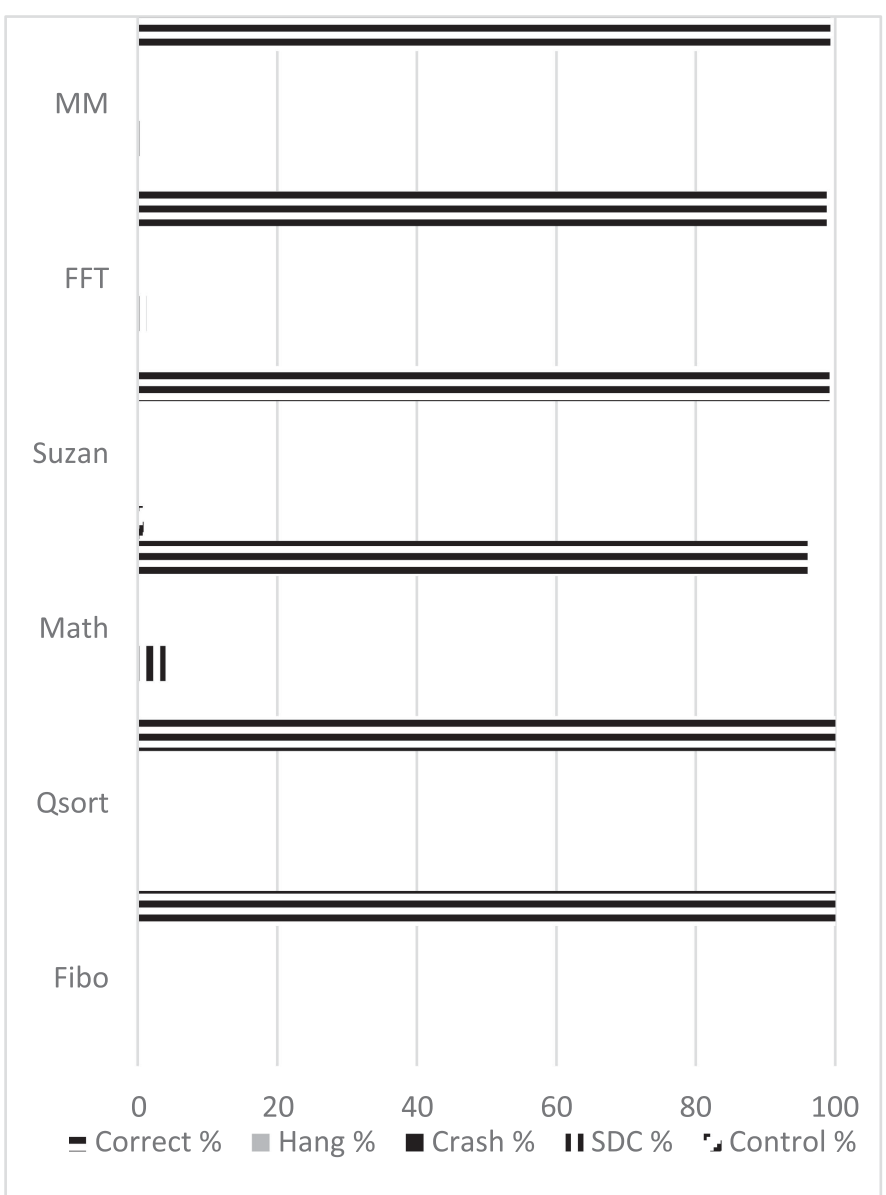

Fig. 14. Protect CPU and memory instructions.

The low error rate achieved using our software protection, was due to the replication of all instruction types (CPU and memory instructions). The fusion of the protection of both instruction types will ensure higher coverage. The datatype formats; i32, i32 $2^{\star}, \mathrm{i} 1, \mathrm{i} 8, \mathrm{i} 8^{\star}$, i64, float and double, float and double pointers have been included in this paper.

We have built our fault injection tool considering single faults to assess SEEs, however we expect that our protection code will detect and recover multiple bit errors, with the condition that they are in the same word. We also can protect multiple, but separate variables, knowing that they are TMR'd using our software. Directly using our protection technique prevented crashes and control errors.

\section{F. ACEDR Time Overhead}

The overhead or the time delay added when applying our protection techniques will be compared to the time delay where no protection is applied. We started by recording the execution time of the unprotected code, after that we protect the code with ACEDR, and then measured its execution time. We used the Linux tool perf, to measure the delays and the number of processor cycles [36]. Table II shows the different time overheads for the different benchmarks protected with ACEDR.
TABLE II

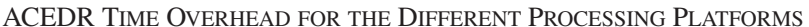

\begin{tabular}{ccc}
\hline \hline Benchmarks & $\begin{array}{c}\text { Intel core i5-3470 } \\
\text { Overhead (\%) }\end{array}$ & $\begin{array}{c}\text { Raspberry Pi 3 } \\
\text { Overhead (\%) }\end{array}$ \\
\hline \hline Fibo & 6.44 & 12.24 \\
Qsort & 13.89 & 16.66 \\
Math & 8.55 & 15.28 \\
Susan & 2.46 & 257.03 \\
FFT & 10.58 & 13.45 \\
MM & 9.39 & 11.63 \\
\hline
\end{tabular}

The study of the time overhead when injecting the code is platform independent (same results on the Raspberry Pi 3 and the Intel core i5-3470 were obtained). The different overheads are depending on the error types, as follows.

1) SDC did not generate any significant overhead (less than $1 \%)$.

2) Hang, produced and indefinite overhead, since the program is stuck in an infinite loop.

3) Control or flow errors have produced significant overhead, which could range from $0 \%$ up to multiple times the original time of execution; in some cases this reached an order of magnitude of the original time. When the overhead is infinity, a hang has occurred.

Crash means that the code did not execute or terminated incorrectly producing $0 \%$ overhead.

The high performance (low time overhead) was thanks to the pipeline, where independent redundant instructions have been executed in parallel. The desktop performance is better than the embedded Raspberry Pi 3 (especially when running Susan benchmark), due to the big difference in the two platforms performances. Another important factor is the compiler optimizations, improving the performance highly. This demonstrates the portability of the ACEDR, and compilation of protected code for differing processing architectures.

\section{G. ACEDR and State-of-the Art}

Compared to the state-of-the-art EDDI [24] and SWIFT [25], where both schemes are used for error detection only without the ability to recover, where all instructions have been duplicated (Memory and CPU instructions for EDDI, partially memory and all CPU instructions for SWIFT), our method offers better performance in terms of the overhead generated.

In addition to this, our method is capable of error detection and recovery as well, which is very important in real-time applications, where the recovery part enables the system to keep running reliably. In the state of the art, the error rate of injection of the baseline code (unprotected) was predetermined (20\% error for EDDI and 37\% for SWIFT) which could impact dramatically the results of injecting the protected code. Our paper does not predetermine the baseline error rate. Every benchmark's instructions are injected independently, which results in different baseline error rates (from $25.06 \%$ up to $87.09 \%$ ). 
This will yield more realistic results, when the protected code is injected.

\section{RELIABILITY COMPARISON OF INJECTION EXPERIMENTS WITH PREDICTIONS}

Section V, where the injection experiments have been performed, has shown that the error rate has been dropped using purely the software protection techniques developed. Since we have obtained the different sensitivities for the different instructions types, we can measure the precision of our reliability prediction model from Section III, where the reliability obtained from (21) and (34) will be compared, this will show the precision of the theoretical reliability prediction model. In this scenario, we take (34) as the ground truth. In order to show that the protection code did not just drop the error rate, but it also improved the total reliability of the whole system, a comparison between (21) and (34) will be done.

The instruction types are divided to two major categories (CPU/Memory), however, the number of instruction types can be subdivided to the total number of types "l" that exists in the benchmark.

The key remarks are as follows.

1) Both the predictions and the injection experiment show an improvement in reliability when protecting using ACEDR.

2) The reliability of the prediction is higher than the reliability of the injection experiment at the start; then both curves intersect. After the intersection point, the reliability of injection becomes higher than the reliability of predictions.

\section{A. Mean Time to Failure (MTTF)}

In order to determine the accuracy of our prediction techniques, compared to the results of the injection, we investigated the MTTF as a key performance metric. By definition, MTTF is the length of time a device or other product is expected to last in its operation. MTTF is obtained by integrating reliability over time as the following: MTTF $=\int_{0}^{\infty} R(t)$. The MTTF of the injection experiment is obtained by integrating (34), and the MTTF of the prediction is obtained by integrating (28).

The accuracy of our predictions is determined by the error between the injected and predicted MTTFs. The residual error is dependent on the error rates of the CPU and the first level of caches $\lambda_{\mathrm{p}}$ and $\lambda_{\mathrm{c}}$, the reason for this is the fact that the hit rate of the first level of cache was more than or equal to $98 \%$ in all of the tested benchmarks. We plot the error as a function of the ratio $\lambda_{\mathrm{p}} / \lambda_{\mathrm{c}}$ we obtained in Fig. 15.

Fig. 15 includes the error of MTTF for the previously injected benchmarks. We note that the lowest errors for the benchmarks are $8.9 \times 10^{-4}, 3.2 \times 10^{-3}, 2.3 \times 10^{-3}, 0.028,8.06 \times 10^{-3}$, and $2.88 \times 10^{-5}$, respectively. We define these as the optimal points. The $\lambda_{\mathrm{p}} / \lambda_{\mathrm{c}}$ ratio error become linear and constant after this point at $0.030,0.042,0.076,0.4,0.7$, and 1.6 , respectively. Errors corresponding to ratios less than the minimal points are high and are considered inaccurate. We would recommend users of our method for future predictions and models if greater than this minima.

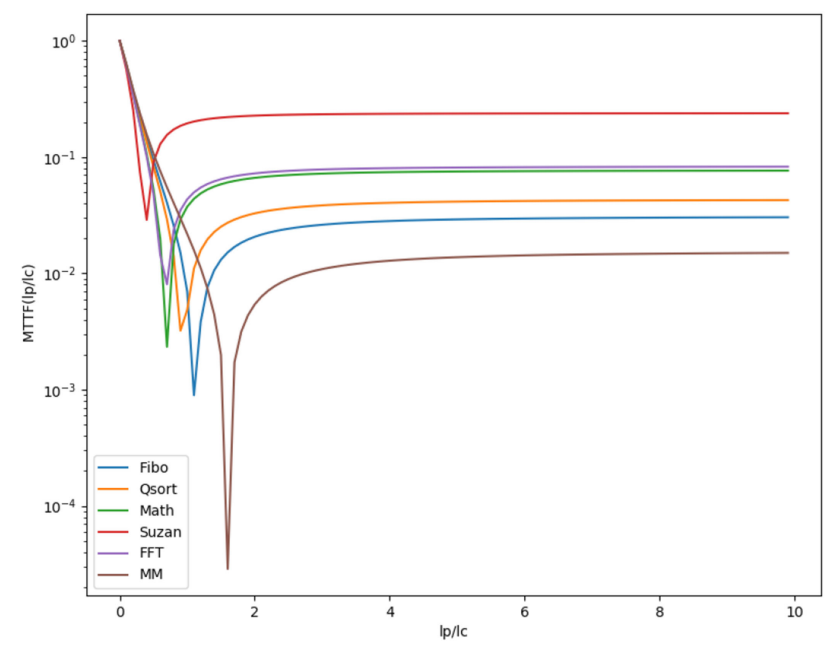

Fig. 15. MTTF error with respect to $\left(\lambda_{\mathrm{p}} / \lambda_{\mathrm{c}}\right)$ ratio.

\section{CONCLUSION}

Bit-flips originating from SEEs are becoming a prominent problem in the processor architectures. It is crucial for designers in both mainstream and embedded or critical processing systems to ensure the reliability of their systems. Systems with redundant hardware that make use of hard-ECC and hard-TMR will elevate the design complexity of terrestrial applications, often eliminating it as an option. Soft error detection and recovery methods are viable alternatives because of their high coverage and low overhead and allowing for the best trade space between reliability and performance, providing engineers with flexible ways of protecting their processing architectures.

New ACEDR techniques were implemented at compiler level in this paper. We implemented and verified a new error injection tool with experiments on different benchmarks in order to test the reliability of our software protection techniques. We injected all instructions of the chosen benchmarks, where we divided the instructions to two main categories; Memory instructions, allowing R/W operations on the cache memory of the processing architecture, and CPU instructions including logic and Arithmetic operations. To quantify our results, we injected both the protected and the unprotected code and compared the results. We showed that CPU registers and their data or instructions can be fully protected against the bit-flips caused by the fault injection experiment simulating SEEs. For both the first and second benchmarks, we demonstrated that all instructions can be fully protected with almost $100 \%$ error injection coverage. When injecting errors in code, we greatly reduced error rates in the benchmarks: from $73.08 \%$ to $0 \%$ for Fibo, from $46.16 \%$ to $0 \%$ for Qsrt, from $87.41 \%$ to $4.45 \%$ for Math, from $25.03 \%$ to $0.83 \%$ for Susan, from $54.12 \%$ to $1.23 \%$ for FFT, and from $59.72 \%$ to $0.71 \%$ for MM.

In the third benchmark (Math) including (SolveCubic, Rad2Deg, Deg2Rad, UQsort), the CPU instructions were fully protected, even though the third benchmark was not fully covered, all crashes and control/flow errors have been eliminated using ACEDR. The high error detection and recovery was due to the replication of multiple data and instruction types, 
including i32, i32 $2^{\star}, \mathrm{i} 1, \mathrm{i} 8, \mathrm{i} 8^{\star}$, i64, float and double, float and double pointers. The ACEDR protection mitigates different error types (crashes, SDC, control/flow), with low time overhead in multicore across multiple platforms, enabling the use of our protection technique to many architectures. We found that the delays measured were not highly significant because of the pipelining of independent redundant instructions, allowing the use of abundant resources of the CPU architectures, without causing a bottleneck.

In this paper, we also showed equations modeling the reliability of the whole processing architecture, using multiple parameters related to the hardware architecture and the environment where the architecture is operating. The injection experiments confirm the reliability predictions, where, for both benchmarks, the curves show that the reliability of the protected code is higher than the reliability of the unprotected one. The precision of our prediction model depends on the value of the initial variables (the error rates of the CPU and the caches); the model can reach very high accuracy level if the ratio $\lambda_{\mathrm{p}} / \lambda_{\mathrm{c}}$ corresponds to the optimal point. For values more than the optimal point, the accuracy will be from 92.35 to $98.5 \%$ depending on the benchmark, except for the Suzan benchmark where the accuracy was 77\%, which is due to the randomness of the error injection in this benchmark. Our model is limited if the values of $\lambda_{p} / \lambda_{c}$ are less than the optimal point, where the MTTF error can reach high rates.

This paper would be highly valuable, both to satellites/space, but also in general computing, such as in in aircraft, automotive, server farms, and medical equipment (or anywhere that needs safety critical performance) as hardware gets smaller and more susceptible.

\section{REFERENCES}

[1] R. C. Baumann, "Soft errors in advanced semiconductor devices-part I: The three radiation sources," IEEE Trans. Device Mater. Rel., vol. 1, no. 1, pp. 17-22, Mar. 2001

[2] T. J. O'Gorman et al., "Field testing for cosmic ray soft errors in semiconductor memories," IBM J. Res. Develop., vol. 40, no. 1, pp. 41-50, 1996.

[3] P. Shivakumar et al., "Modeling the effect of technology trends on the soft error rate of combinational logic," in Proc. Int. Conf. Dependable Syst. Netw., 2002, pp. 389-398.

[4] P. Pukite and J. Pukite, Markov Modeling for Reliability Analysis. WileyIEEE Press, 1998.

[5] R. Ginosar, "Survey of processors for space," Data Syst. Aerosp. (DASIA). Eurospace, pp. 1-5, 2012.

[6] R. W. Horst, R. L. Harris, and R. L. Jardine, "Multiple instruction issue in the NonStop cyclone processor," in Proc. 17th Annu. Int. Symp. Comput. Architecture, 1990, pp. 216-226.

[7] T. J. Slegel et al., "IBM's S/390 G5 microprocessor design," IEEE Micro, vol. 19, no. 2, pp. 12-23, Mar./Apr. 1999.

[8] M. Tremblay and Y. Tamir, "Support for fault tolerance in VLSI processors," in Proc. IEEE Int. Symp. Circuits Syst., 1989, pp. 388-393.

[9] R. Phelan, "Addressing soft errors in ARM core-based SoC," ARM, Cambridge, U.K., White Paper, 2003.

[10] Y. Nezzari and C. Bridges, "Compiler extensions towards reliable multicore processors," in Proc. IEEE Aerosp. Conf., 2017, pp. 1-6.

[11] C. Lattner and V. Adve, LLVM Language Reference Manual, 2006.

[12] A. Avizienis et al., "Basic concepts and taxonomy of dependable and secure computing," IEEE Trans. Dependable Secure Comput., vol. 1, no. 1, pp. 11-33, 2004.

[13] N. Aggarwal et al., "Configurable isolation: Building high availability systems with commodity multi-core processors," in ACM SIGARCH Comput. Architecture News, vol. 35, pp. 470-481, 2007.
[14] A. Vajda, "Multi-core and many-core processor architectures," in Programming Many-Core Chips. Springer, 2011, pp. 9-43.

[15] J. P. Walters et al., "Software-based fault tolerance for the maestro manycore processor," in Proc. IEEE Aerosp. Conf., 2011, pp. 1-12.

[16] A. Avizienis, "The N-version approach to fault-tolerant software," IEEE Trans. Softw. Eng., vol. 1985, no. 12, pp. 1491-1501, Dec. 1985.

[17] K. Mitropoulou, V. Porpodas, and M. Cintra, "DRIFT: Decoupled compiler-based instruction-level fault-tolerance," in International Workshop on Languages and Compilers for Parallel Computing. Springer, 2013.

[18] J. E. Fritts et al., "MediaBench II video: Expediting the next generation of video systems research," Microprocessors Microsystems, vol. 33, no. 4, pp. 301-318, 2009.

[19] J. L. Henning, "SPEC CPU2000: Measuring CPU performance in the new millennium," Computer, vol. 33, no. 7, pp. 28-35, 2000.

[20] A. Piotrowski, "Automatic installation of software-based fault tolerance algorithms in programs generated by GCC compiler," Int. J. Microelectronics Comput. Sci., vol. 1, no. 3, pp. 263-268, 2010.

[21] G. A. Reis, J. Chang, and D. I. August, "Automatic instruction-level software-only recovery," IEEE Micro, vol. 27, no. 1, pp. 36-47, Jan./Feb. 2007.

[22] S. Feng et al., "Shoestring: Probabilistic soft error reliability on the cheap," in ACM SIGARCH Comput. Architecture News, vol. 38, pp. 385-396, 2010

[23] J. Yu and M. J. Garzaran, "Compiler optimizations for fault tolerance software checking," in Proc. 16th Int. Conf. Parallel Architecture Compilation Techn., 2007, p. 433.

[24] N. Oh, P. P. Shirvani, and E. J. McCluskey, "Error detection by duplicated instructions in super-scalar processors," IEEE Trans. Rel., vol. 51, no. 1 , pp. 63-75, Mar. 2002.

[25] G. A. Reis et al., "SWIFT: Software implemented fault tolerance," in Proc. Int. Symp. Code Gener. Optim., 2005, pp. 243-254.

[26] UIUC Open IMPACT Effort, "The OpenIMPACT IA-64 compiler.

[27] Y.Zhang et al., "DAFT: Decoupled acyclic fault tolerance," Int. J. Parallel Program., vol. 40, no. 1, pp. 118-140, 2012.

[28] C. Wang et al., "Compiler-managed software-based redundant multithreading for transient fault detection," in Proc. Int. Symp. Code Gener. Optim., 2007, pp. 244-258.

[29] K. Mitropoulou, V. Porpodas, and M. Cintra. "CASTED: Core-adaptive software transient error detection for tightly coupled cores," in Proc. IEEE 27th Int. Symp. Parallel Distrib. Process., 2013, pp. 513-524.

[30] R. M. Stallman, "Using and porting the GNU compiler collection," Free Softw. Found., vol. 51, pp. 02110-1301, 1989.

[31] U. Afzaal and J.-A. Lee, "A self-checking TMR voter for increased reliability consensus voting in FPGAs," IEEE Trans. Nuclear Sci., vol. 65, no. 5, pp. 1133-1139, May 2018.

[32] Y. Nezzari and C. Bridges, "Modelling processor reliability using LLVM compiler fault injection," in Proc. IEEE Aerosp. Conf., 2018, pp. 1-10.

[33] LLVM, LLVM Language Reference Manual. (2018). [Online]. Available: https://llvm.org/docs/LangRef.html

[34] LLVM, LLVM IR builder. (2018). [Online]. Available: http://llvm.org/doxygen/classllvm_1_1IRBuilder.html

[35] M. Consortium, MediaBench II benchmark. (2015). [Online]. Available: http://mathstat.slu.edu/ fritts/mediabench

[36] A. C. de Melo, "The new linux'perf'tools," in Proc. Slides Linux Kongress, 2010, p. 18.

Yasser Nezzari received the State Engineering degree in control engineering from the Institute of Electrical and Electronic Engineering at the University of Boumerdes, Boumerdes, Algeria, in 2014. He is currently working toward the Ph.D. degree at the Surrey Space Centre, University Of Surrey, Guildford, U.K., where he is involved in researching software compilers, radiation tolerance using software, and adaptive software.

Christopher P. Bridges received the B.Eng. degree from the University of Greenwich, London, U.K., in 2005, and the Ph.D. degree from the University of Surrey, Guildford, U.K., in 2009, both in electronic engineering.

In 2013, he designed, built, and still operates the U.K.'s first CubeSat (STRaND-1) with Surrey Satellite Technology (SSTL) and now contributes toward computing hardware and software with SSTL, on ESA's ESEO mission and also the NASA-JPL/CalTech AAReST mission. He currently leads the On-Board Data Handling (OBDH) research group within Surrey Space Centre, University Of Surrey. His research interests include software-defined radios, real-time embedded systems, agent computing, Java processing, multicore processing in FPGAs, and astrodynamic computing methods in many spaceflight payloads. 